\title{
The Impact of Interactive Manipulation on the Recognition of Objects
}

\author{
Frank Meijer ${ }^{\mathrm{a}}$, Egon L. van den Broek ${ }^{\mathrm{b}}$ and Theo Schouten ${ }^{\mathrm{c}}$ \\ ${ }^{a}$ Dept. of Cognitive Psychology and Ergonomics, Faculty of Behavioural Sciences, \\ University of Twente \\ P.O. Box 217, 7500 AE Enschede, The Netherlands \\ f.meijer@utwente.nl \\ ${ }^{\mathrm{b}}$ Center for Telematics and Information Technology (CTIT), University of Twente \\ P.O. Box 217, 7500 AE Enschede, The Netherlands \\ vandenbroek@acm.org http://eidetic.ai.ru.nl/egon/ \\ Institute for Information Science (ICIS), Radboud University Nijmegen \\ P.O. Box 9104, 6500 HE Nijmegen, The Netherlands \\ t.schouten@cs.ru.nl \\ http://www.cs.ru.nl/ths/
}

\begin{abstract}
A new application for VR has emerged: product development, in which several stakeholders (from engineers to end users) use the same VR for development and communicate purposes. Various characteristics among these stakeholders vary considerably, which imposes potential constraints to the VR. The current paper discusses the influence of three types of exploration of objects (i.e., none, passive, active) on one of these characteristics: the ability to form mental representations or visuo-spatial ability (VSA). Through an experiment we found that all users benefit from exploring objects. Moreover, people with low VSA (e.g., end users) benefit from an interactive exploration of objects opposed to people with a medium or high VSA (e.g. engineers), who are not sensitive for the type of exploration. Hence, for VR environments in which multiple stakeholders participate (e.g. for product development), differences among their cognitive abilities (e.g., VSA) have to be taken into account to enable an efficient usage of VR.
\end{abstract}

Keywords: Interactive exploration, mental representations, visuo-spatial ability

\section{INTRODUCTION}

In the past two decades, Virtual Reality (VR) has emerged as a powerful technology that can simulate environments for professional purposes. VR has become more and more accessible and its technology is successfully applied in numerous professions, such as medicine, education, art and entertainment, and the military. Furthermore, new application areas arise, such as product creation and realization; e.g., [1] [2] [3]. Within the SenterNovem project Synthetic Environments, the use of VR is exploited for product design. VR enables different stakeholders (e.g., end users or engineers) to experience a product long before the phase of physical prototyping. In the project, the usage of simulated products becomes an advantage when the construction of VR is time and cost efficient. Instead of simulating entire virtual worlds, only a limited set of features of VR is integrated in the simulation. The current research will examine what features will contribute to a sufficient product experience and why. In particular, the influence of two specific features on human cognition will be the topic of research: multimodality and interactivity. The present study will focus on the latter.

Wohlschlager and Wohlschlager [4] provided evidence that in human cognition, motor processes and mental representations are interrelated. They based their study on the mental rotation task of Shepard and Metzler [5] in which participants compare two similar objects. Both objects are viewed from different angles and the participants are required to mentally rotate one of these objects to make a decision whether or not it is the same. The time to make this decision increases linearly with the angular difference of the two objects. Wohlschlager and Wohlschlager found that if participants manually rotated an object to match it with another, their performance time increased at the same rate as when they mentally rotated the objects. Furthermore, when the participants manually rotated an object while doing a mental rotation task, interference on performance occurred when the manual and mental rotations were done over

Human Vision and Electronic Imaging XIII, edited by Bernice E. Rogowitz, Thrasyvoulos N. Pappas,

Proc. of SPIE-IS\&T Electronic Imaging, SPIE Vol. 6806, 68060H, (C 2008 SPIE-IS\&T · 0277-786X/08/\$18 
different axes. Harman et al. [6] showed that interactivity improves the development of mental representations of objects. In their study, participants were instructed to study a set of computer-mediated 3D objects by either active manipulation or passive observation. Afterwards, they examined to what extend an object was mentally represented in a recognition test. In this test, a 2D image of an object was presented on which a decision was made whether or not this objects was previously studied. Harman et al. found that participants, who had first interactively studied an object, were faster in their decisions than who had passively observed the object. In 2001, James et al. [7] confirmed these results when participants made their decisions on a mental rotations task.

We conducted an experiment in the line of Harmann et al. (1999) and James et al. (2001) to investigate the influence of interactivity for people with differing characteristics. In product development a wide range of stakeholders are involved who considerably differ in their characteristics such as their visuo-spatial ability (VSA); i.e., the capacity to construct and manipulate mental representations of objects and environments. We examined whether the influence of three types of exploration of objects (i.e., none, passive, and active) on mental representations, depends on the people's VSA. People differ in this ability: Some have great difficulty imagining and mentally manipulating a visual spatial image whereas others have not. We hypothesize that: 1) interactive manipulation contributes to the development of mental representations in general, and 2) people with low VSA benefit more from interactive manipulation than those with high VSA. The experimental set-up will be described in the next section followed by the results and a discussion of the implications it has on the development of VR.

\section{MATERIALS AND METHODS}

\subsection{Participants}

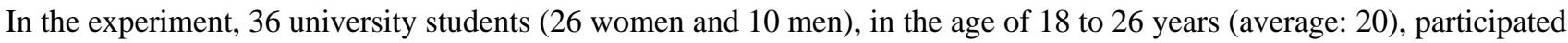
in exchange for course credits. All participants had normal or corrected to normal visual acuity and no known neurological or visual disorders.

\subsection{Materials and apparatus}

A set of 48 novel non-symmetrical three-dimensional objects was created in the 3D modeling program Art of Illusion (Free Software Foundation, Inc.). The objects were constructed from a set of 24 "geon-like" components [8]. Each object consisted of a big center component with smaller components directly attached to it. In total, 24 "simple" objects, consisting of three components, and 24 "difficult" objects, consisting of five components, were created. The objects were colored gray and were equal in illumination and luminance. These 3D objects were used both as study objects and test objects in respectively the study phase and the test phase of the experiment (see also Figure 1).

Two desktop computers were used in the experiment. 1) A Pentium IV computer running Authorware 7.01 (Macromedia, Inc.). The presentation of the study objects was enabled by the Cortona VRML Client 5.1 (Parallel Graphics, Inc.) plug-in, using the ActiveX option in Authorware. 2) A Pentium II computer running E-Prime 1.1 (Psychology Software Tools, Inc.) acquired the necessary response data through the standard keyboard. Both computers were connected to identical calibrated 17" Philips 107-T5 $60 \mathrm{~Hz}$ monitors (see Figure 2). The study objects were equal in size and were presented within an imaginary circle of $20 \mathrm{~cm}$ in diameter on the center of the screen. The test objects were equal in size presented within an imaginary circle of $10 \mathrm{~cm}$ in diameter. These test objects were presented simultaneously with a distance of $8 \mathrm{~cm}$ left and right from the center of the screen. All objects were presented on a gray background $\left(184.0 \mathrm{~cd} / \mathrm{m}^{2}\right)$ at a viewing distance of $60 \mathrm{~cm}$. 

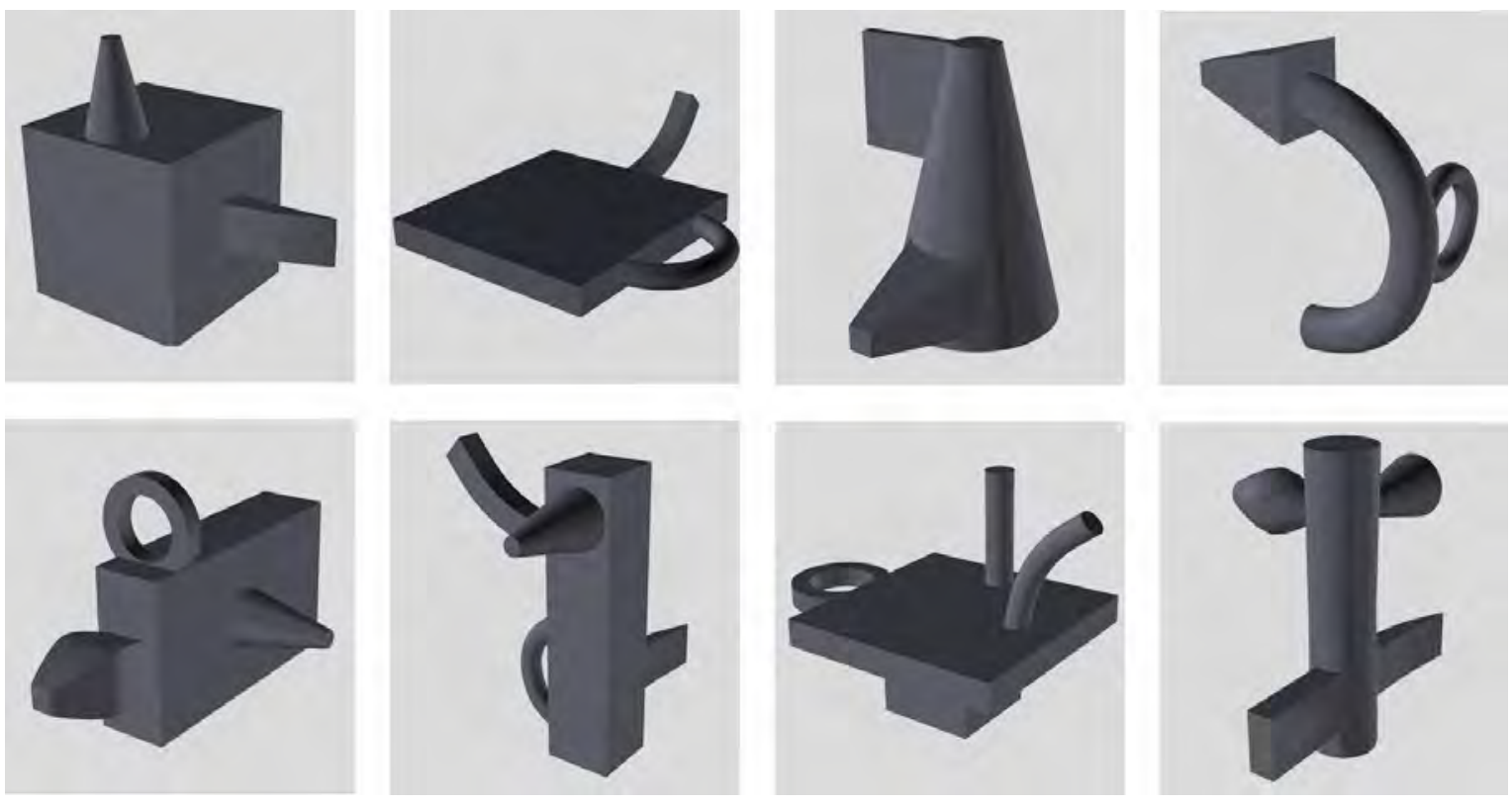

Fig. 1. Examples of simple objects (top row) and difficult objects (bottom row) as constructed for and used in the experiment, with respectively three and five geons.

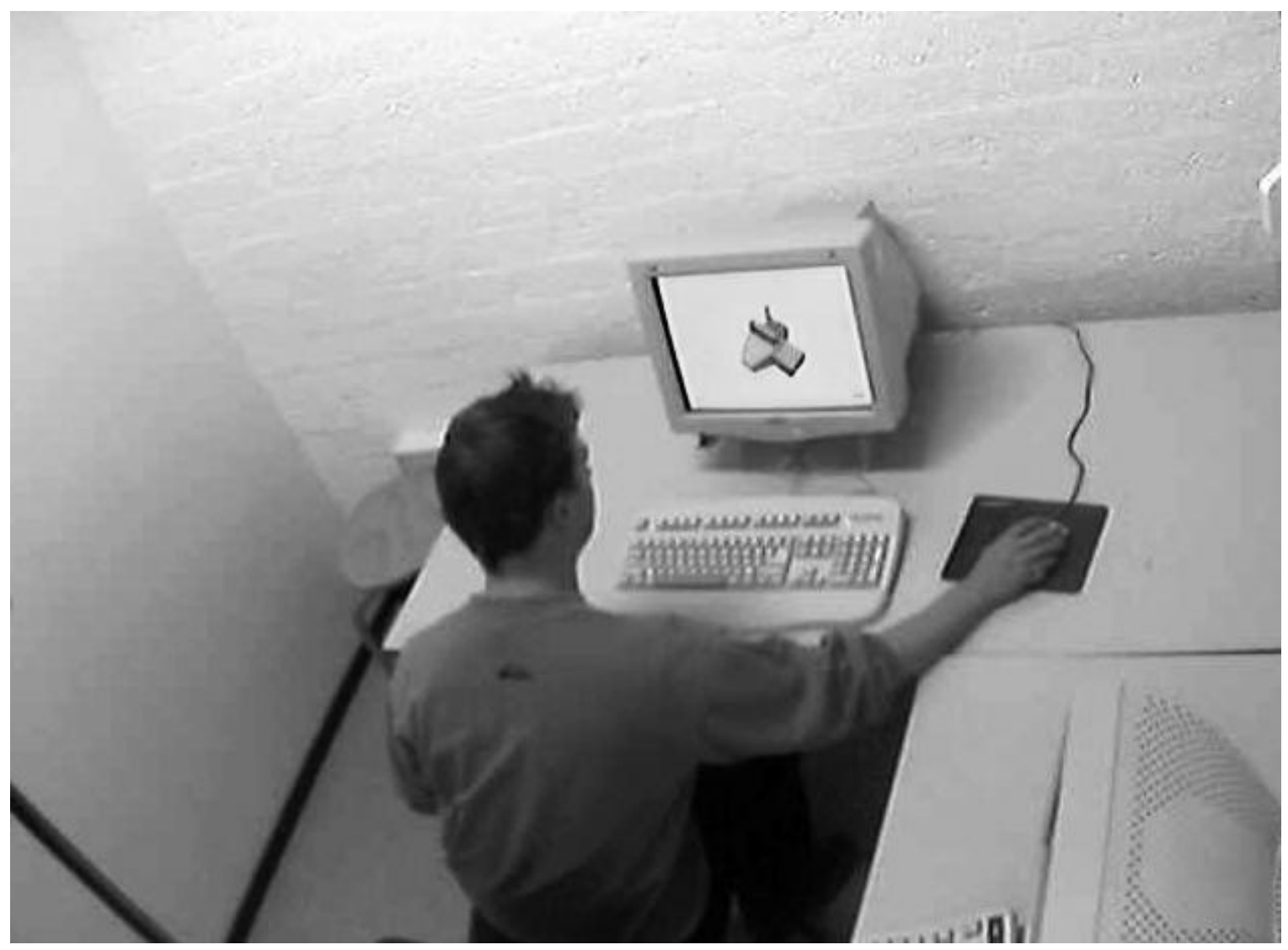

Fig. 2. The two desktop computers as used in the experiment. Participants switched from one computer to the other between the study phase and the test phase. 


\subsection{General procedure and design}

Before the experiment started, participants were tested on their visual-spatial ability (VSA), using a mental rotations test [9]. Based on their results, the participants were divided into three groups of VSA: low, medium, and high. After this pretest, the experiment started with 2 practice trials followed by $3 \times 4$ blocks of experimental trials. Each block comprised a study phase and a test phase, each presented on another computer, between which the participants switched in each block (see Table 1).

Table 1. The experiment consisted of 3 blocks of conditions: control $(\mathrm{C})$ in which a simple math task was executed, passive $(\mathrm{P})$, and active $(\mathrm{A})$ exploration of objects. The order of blocks $(\mathrm{C}, \mathrm{P}$, and $\mathrm{A})$ was counterbalanced. Each block consisted of 4 study phases $(\mathrm{S})$ and 4 test phases $(\mathrm{T})$, which were presented on two different computers. In total, 32 objects were studied and 48 objects were tested.

\begin{tabular}{|c|c|c|c|c|c|c|c|c|c|c|c|c|c|c|c|c|c|c|c|c|c|c|c|c|}
\hline Block & \multicolumn{2}{|c|}{ C1 } & \multicolumn{2}{|c|}{ C2 } & \multicolumn{2}{|c|}{ C3 } & \multicolumn{2}{|c|}{$\mathrm{C} 4$} & \multicolumn{2}{|c|}{ P5 } & \multicolumn{2}{|c|}{ P6 } & \multicolumn{2}{|c|}{ P7 } & \multicolumn{2}{|c|}{ P8 } & \multicolumn{2}{|c|}{ A9 } & \multicolumn{2}{|c|}{ A10 } & \multicolumn{2}{|c|}{ A11 } & \multicolumn{2}{|c|}{ A12 } \\
\hline Procedure & $\mathrm{S}$ & $\mathrm{T}$ & S & $\mathrm{T}$ & $\mathrm{S}$ & $\mathrm{T}$ & $\mathrm{S}$ & $\mathrm{T}$ & $\mathrm{S}$ & $\mathrm{T}$ & $\mathrm{S}$ & $\mathrm{T}$ & $\mathrm{S}$ & $\mathrm{T}$ & $\mathrm{S}$ & $\mathrm{T}$ & $\mathrm{S}$ & $\mathrm{T}$ & $\mathrm{S}$ & $\mathrm{T}$ & $\mathrm{S}$ & $\mathrm{T}$ & $\mathrm{S}$ & $\mathrm{T}$ \\
\hline Objects & $\mathrm{x}$ & $\begin{array}{l}1 \\
\ldots\end{array}$ & $\mathrm{x}$ & $\begin{array}{l}5 \\
\ldots \\
8\end{array}$ & $\mathrm{x}$ & $\begin{array}{l}9 \\
\ldots \\
12\end{array}$ & $\mathrm{x}$ & $\begin{array}{l}13 \\
\ldots \\
16\end{array}$ & $\begin{array}{l}17 \\
\ldots \\
20\end{array}$ & $\begin{array}{l}17 \\
\ldots \\
20\end{array}$ & $\begin{array}{l}21 \\
\ldots \\
24\end{array}$ & $\begin{array}{l}21 \\
\ldots \\
24\end{array}$ & $\begin{array}{l}25 \\
\ldots \\
28\end{array}$ & $\begin{array}{l}25 \\
\ldots \\
28\end{array}$ & $\begin{array}{l}29 \\
\ldots \\
32\end{array}$ & $\begin{array}{l}29 \\
\ldots \\
32\end{array}$ & $\begin{array}{l}33 \\
\ldots \\
36\end{array}$ & $\begin{array}{l}33 \\
\ldots \\
36\end{array}$ & $\begin{array}{l}37 \\
\ldots \\
40\end{array}$ & $\begin{array}{l}37 \\
\ldots \\
40\end{array}$ & $\begin{array}{l}41 \\
\ldots \\
44\end{array}$ & $\begin{array}{l}41 \\
\ldots \\
44\end{array}$ & $\begin{array}{l}45 \\
\ldots \\
48\end{array}$ & $\begin{array}{l}45 \\
\ldots \\
48\end{array}$ \\
\hline
\end{tabular}

\subsection{Study phase procedure}

The experiment started with a study phase in which participants studied the objects in three exploration conditions (see Figure 3). In control conditions, participants conducted a simple math task and no objects were studied, which provided a baseline of the participant's performance for the test phase. In passive exploration conditions, participants did not interact with the object but observed it while rotating $360^{\circ}$ over the $\mathrm{x}$-axis, $\mathrm{y}$-axis, and z-axis. In active exploration conditions, participants were free to rotate the object in any direction with a computer mouse. The order of exploration blocks was counterbalanced. Each block consisted of two simple and two difficult objects, randomly selected from the set of 48 objects, with the order of object difficulty counterbalanced. Each object was studied for 30 seconds with a 5 second interval between them.
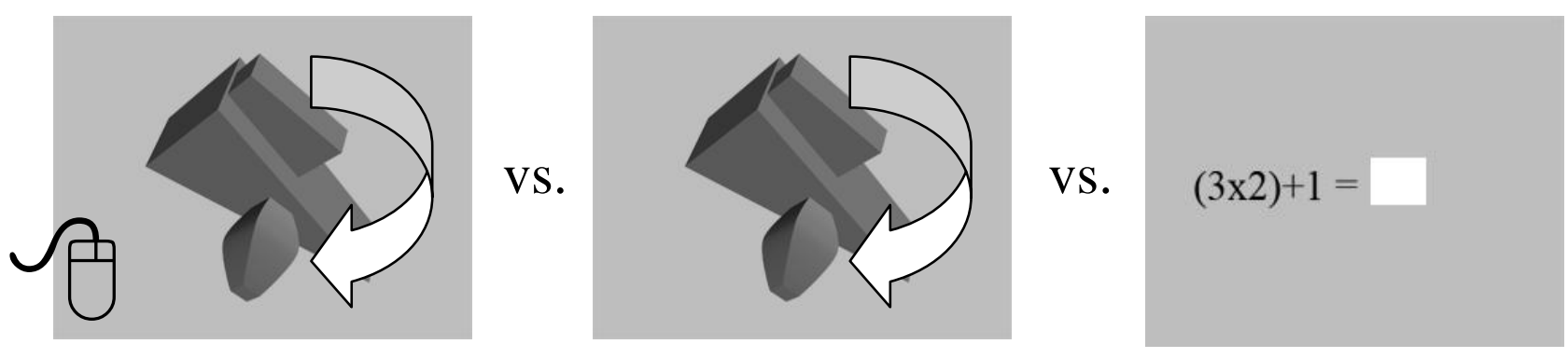

Fig. 3. Schematic overview of the different exploration conditions. The left and middle images represent the active and passive conditions respectively. The left image represents the control condition, in which participants were required to conduct a simple math task.

\subsection{Test phase procedure}

After the study phase, participants were instructed to switch to another computer for a test phase. Each trial in the test phase started with a 750 msec presentation of a black fixation cross in the center of the screen followed by two test objects presented simultaneously on the screen (see Figure 4). These objects were 2D images, shown with all components visible. The object displayed left on the screen was always the object as presented in the study phase. The object displayed on the right was always the target object. This target object was either the same as or perfectly mirrored 
compared to the original object. In addition, the target object was $180^{\circ}$ rotated over the $\mathrm{x}$-axis, $\mathrm{y}$-axis, or z-axis. When the test objects appeared, the participants were required to determine as quickly and accurately as possible whether or not these objects were the same. The test objects remained visible until a key-response was given: $\mathrm{m}$ for same object, $\mathrm{z}$ for mirrored object. Response latency and accuracy were recorded. This procedure continued until responses on all four different target objects were given. Each of these objects appeared three times as target object rotated $180^{\circ}$ over one of its axes, both as the same and mirrored version. Consequently, 24 different target objects presented in each test block and a total of 268 responses were given in the complete experiment.
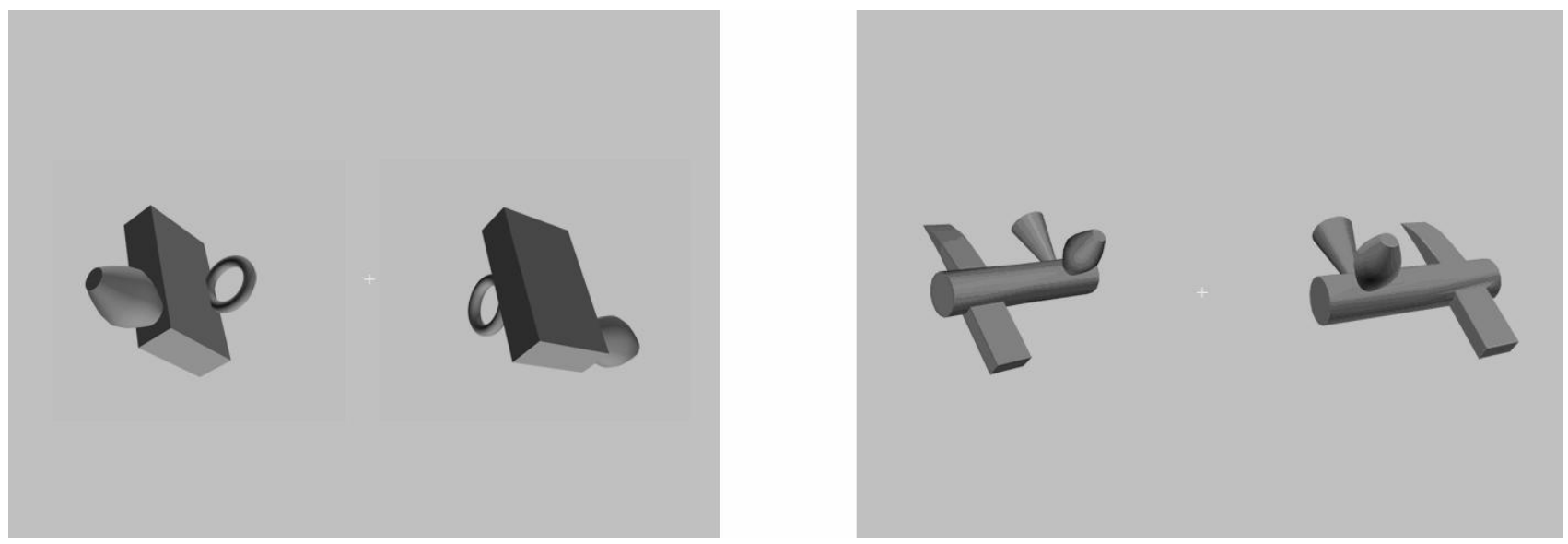

Fig. 4. Screenshots of the displays as shown in the test phase. The left display depicts rotated objects, and the right display mirrored objects. Participants were required to respond as fast and as accurate as possible whether these objects were the same objects or not.

\section{RESULTS}

Two separate repeated-measures 3x2 (i.e., active, passive, and control condition versus simple and difficult object condition) ANOVAs were run with VSA group as between-subjects factor, one for the accuracy data and one for the reaction times. Trials were discarded per block in each condition (i.e., exploration and object complexity condition) in which reaction times exceeded three times the standard deviation. This procedure was executed two times. In total, 2 percent of the total data was excluded from our analyses.

The ANOVA revealed a main effect in the accuracy data of the three exploration conditions $(F(2,35)=8.67, \mathrm{p}<.001)$. Paired comparisons revealed an effect of the control condition compared to the other two conditions $(F(1,35)=12.50$, $\mathrm{p}$ $=.001)$. Participants were more accurate in their decisions after active exploration $(M=79.7$ percent correct answers) and passive observation of objects $(M=78.8)$ than not having explored objects $(M=74.9)$ (see also Figure 5). Paired comparisons between active and passive exploration did not show a significant effect. However, there was a significant interaction effect between exploration condition (passive/active) and VSA group $(F(2,35)=3.36, \mathrm{p}=.047)$. Only the low VSA group showed a significant effect of exploration condition $(F(1,35)=7.64, \mathrm{p}<.018)$, the others did not. Only the low VSA group benefited from active exploration $(M=75.5)$ compared to passive observation $(M=71.0)$ (see Figure 6). Furthermore, participants were more accurate comparing simple objects $(M=79.7)$ than difficult objects $(M=75.9)$ $(F(1,35)=17.99, \mathrm{p}<.001)$. Analysis of the reaction times data revealed no significant main effect of exploration conditions. However, there was a main effect of object complexity $(F(1,35)=299.47, \mathrm{p}<.001)$. Participants were significantly faster making decisions on simple objects $(M=3,915 \mathrm{~ms})$ than on difficult objects $(M=4,857 \mathrm{~ms})$. 


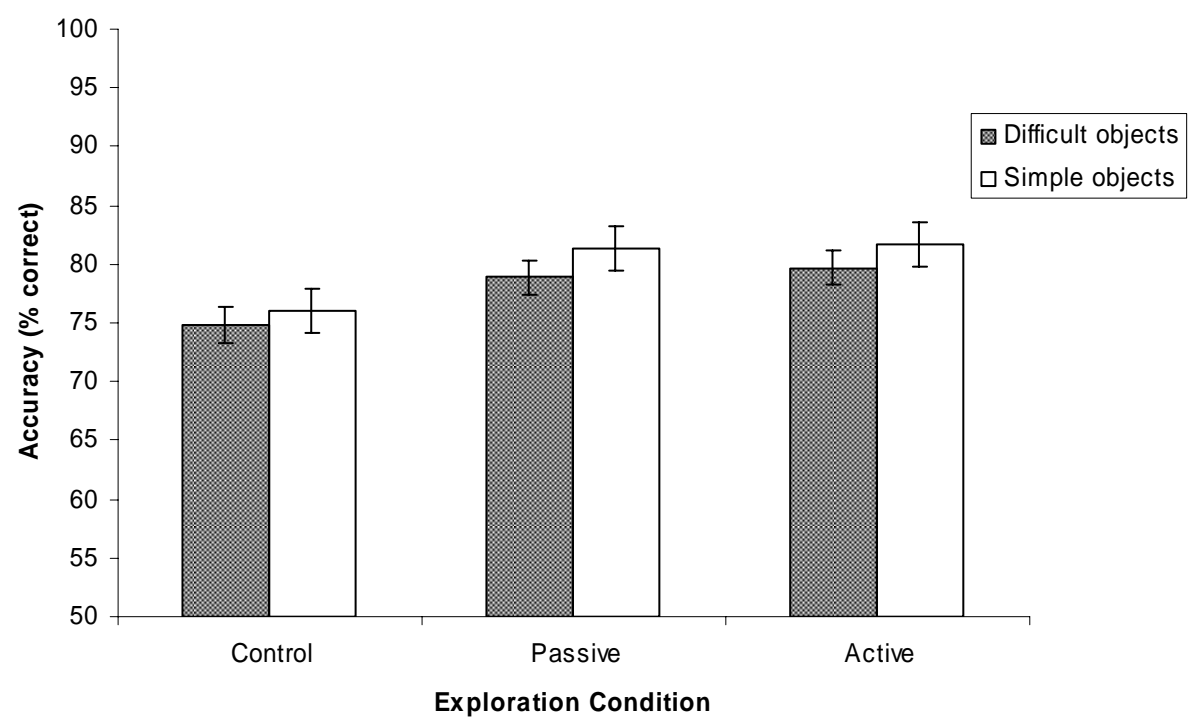

Fig. 5. The percentage of correctly identified target objects during the test phase for each exploration condition. Participants were significantly more accurate in the passive and active conditions than in the control conditions and were more accurate with simple objects than with difficult objects. Note that a mean score of 50 percent is a mere chance level. Error bars indicate one standard deviation above and below the mean.

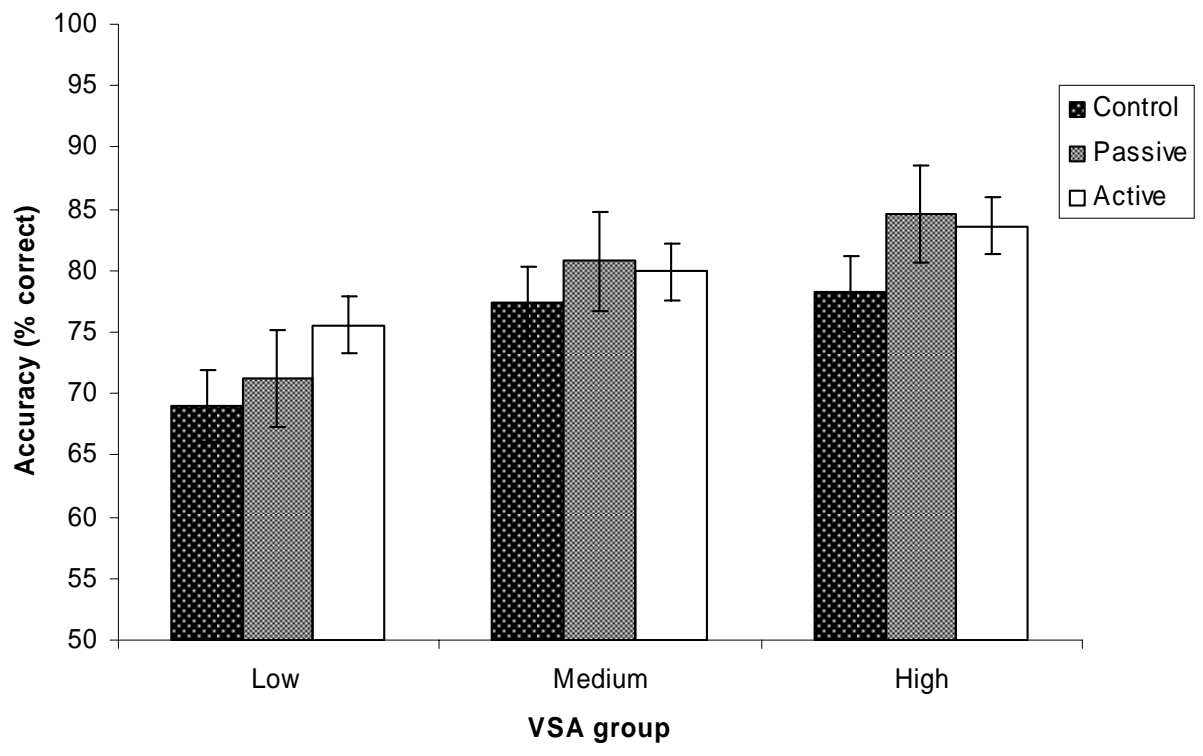

Fig. 6. The percentage of correctly identified target objects during the test phase for each VSA group. There was only a significant difference between the passive and active explorations condition for the low VSA group. Error bars indicate one standard deviation above and below the mean.

\section{DISCUSSION AND CONCLUSIONS}

User experiences indicate that merely a technology push is not sufficient for the successful exploitation of Virtual Reality (VR). Various problems arise, especially when different types of users or stakeholders use the same VR or communicate using it. In product development, a wide range of stakeholders is involved, from engineers to end users. The intensified cooperation among these groups throughout the design process stresses the need for new communication 
techniques. For this purpose, VR techniques are applied. Various stakeholders are facilitated to collaborate by simulating products in an early stage of the design process. However, it is questionable whether all the stakeholders, with their different backgrounds and characteristics, experience the VR in the same way. The current paper discusses the influence of a typical VR feature, interactivity, on one specific cognitive characteristic: the ability to form mental representations or visuo-spatial ability (VSA). Through an experiment we found that 1) all users benefit from exploring objects threedimensionally and 2) only those users with a low VSA (e.g., end users) benefit from an interactive exploration of objects opposed to people with a medium or high VSA (e.g., engineers), who are not sensitive for the type of exploration.

The results presented are only partly in line with earlier findings of Harmann et al. (1999) and James et al. (2001), who did find that active exploration leads to more effective mental representations than passive observation in general, where we only found this for participants with a low VSA. The inconsistence with these studies can be explained as followed: In the passive conditions, a study object is rotated $360^{\circ}$ over each of its axes while in active conditions the object's rotation was controlled by the participant. Consequently, it is possible that at least some participants viewed the study object from more angles in the passive conditions than in the active conditions. For future research, we suggest to tackle this problem by presenting the same number of view angles of the object in both conditions.

This research's findings illustrate that 3D exploration helps users to increase their comprehension of objects within the scope of product development but also in general. Especially, people with a low VSA benefit from the intense, interactive usage VR offers. Moreover, the current research emphasizes the relevance of further development of VR techniques. It was initialized from a relatively new domain of VR: product development. It also illustrates the importance of the user's cognitive characteristics; e.g., the ability to form mental representations. Significant differences among people deliver possible constraints to VR and we stress that they are taken into account where possible. Altogether, we hope this research helps in positioning the factor cognition central in VR development since VR is meant to be interactive. It is not only the technological solutions but also the effective involvement of its users that make VR successful.

\section{KNOWLEDGEMENTS}

The authors gratefully acknowledge the support of the Dutch Innovation Oriented Research Program 'Integrated Product Creation and Realization (IOP-IPCR)' of the Dutch Ministry of Economic Affairs.

\section{REFERENCES}

[1] Weyrich, M, and Drews, P., “An interactive environment for virtual manufacturing: the virtual workbench,” Computers in Industry 38(1), 5-15 (1999).

[2] Dangelmaier, W, Fischer, M., Gausemeier, J., Grafe, M., Matyscok, C., and Mueck, B., "Virtual and augmented reality support for discrete manufacturing system simulation,” Computers in Industry 56(4), 371-383 (2005).

[3] Mujber, T.S., Szecsi T., and Hashmi, M.S.J, “Virtual reality applications in manufacturing process simulation,” Proc. International Conference on Advances in Materials and Processing Technologies: Part 2 155-156, 1834-1838 (2004).

[4] Wohlschlager, A., \& Wohlschlager, A., "Mental and manual rotation,” Journal of Experimental Psychology: Human Perception and Performance 24(2), 397-412 (1998).

[5] Shepard, R. N., \& Metzler, J., “Mental rotation of three-dimensional objects,” Science 171, 701-703 (1971).

[6] Harman, K. L., Humphrey, G. K., \& Goodale, M. A., "Active manual control of object views facilitates visual recognition,” Current Biology 9, 1315-1318 (1999).

[7] James, K.H., Humphrey, K., Goodale, M.A., "Manipulating and recognizing virtual objects: where the action is,” Canadian Journal of Experimental Psychology 55(2), 111-120 (2001).

[8] Biederman, I., "Recognition by components: A theory of human image understanding,” Psychological Review 94, 115-147 (1987).

[9] Vandenberg, S.G., Kuse, A.R., “Mental rotations, a group test of three-dimensional spatial visualization,” Perceptual and Motor Skills 47, 599-601 (1978). 